\title{
Organ donation from intensive care units in England
}

\author{
Sheila M Gore, Charles J Hinds, Annabel J Rutherford
}

\begin{abstract}
Objective-To audit all deaths in intensive care units (excepting coronary care only and neonatal intensive care units) in England to assess potential for organ procurement.

Design-An audit in which 14 regional health authorities and London special health authorities each designated a regional liaison officer to identify intensive care units and liaise with Department of Health and the Medical Research Council's biostatistics unit in distribution, return, and checking of audit forms. Audit took place from 1 January to 31 March 1989 and will continue to 31 December 1990.
\end{abstract} Setting - 278 Intensive care units in England.

Participants-Colleagues in intensive care units (doctors, nurses, coordinators, and others), who completed serially numbered audit forms for all patients who died in intensive care.

Results-The estimated number of deaths in intensive care units was 3085 , and validated audit forms were received for 2853 deaths (92\%). Brain stem death was a possible diagnosis in only $407(14 \%)$ patients (about 1700 cases a year) and was confirmed in $282(10 \%)$ patients (an estimated 1200 cases a year). Half the patients ( $95 \%$ confidence interval $45 \%$ to $57 \%$ ) in whom brain stem death was confirmed became actual donors of solid organs. Tests for brain stem death were not performed in $106(26 \%)$ of 407 patients with brain stem death as a possible diagnosis, and general medical contraindication to organ donation was recorded for $48(17 \%)$ of 282 patients who fulfilled brain stem death criteria before cessation of heart beat. The criteria were fulfilled before cessation of heart beat and in the absence of any general medical contraindication to organ donation in 234 patients, $8 \%$ of those dying in intensive care (an estimated 1000 cases a year). Consent for organ donation was given in $152(70 \%)$ of 218 cases $(64 \%$ to $76 \%)$ when the possibility of organ donation was suggested to relatives. In only 14 out of 232 families $(6 \% ; 3 \%$ to $9 \%)$ was there no discussion of organ donation with relatives. Corneal suitability was recorded as "not known" in a high proportion $(1271 ; 45 \%)$ of all deaths and intensive care units reported only 123 corneal donors $(4 \%$ of all audited deaths).

Conclusion - When brain stem death is a possible diagnosis tests should always be carried out for confirmation. Early referral to the transplant team or coordinator should occur in all cases of brain stem death to check contraindications to organ donation. There should be increased use of asystolic kidney donation, and patients should be routinely assessed for suitability for corneal donation. Finally, more publicity and education are necessary to promote consent.

Intensive Care Unit, St Bartholomew's Hospital, London

Charles J Hinds, FCANAES, consultant

Correspondence to: Dr Gore. per million population for retrieving kidneys from cadaver donors were similar internationally: 29 for the United Kingdom Transplant Service and Eurotransplant and 32 for the United States. ${ }^{2}$ But the supply of donor organs for transplantation still fails to meet demand. More than 3700 patients in the United Kingdom were awaiting kidney transplants and nearly 600 heart transplants in 1988, while Eurotransplant's kidney waiting list had increased by $10 \%$ from 8268 in 1987 to 9086 at 31 December 1988. The projected need for kidneys in the United Kingdom is at least 2500 a year and could rise as high as $4000 .{ }^{3}$ Most solid organs (kidneys, heart, liver, lungs, pancreas) are obtained from brain stem dead donors. In 1981 Jennett estimated that 4000 brain stem deaths occurred annually in the United Kingdom, of whom only about a sixth became actual donors. ${ }^{4}$ This led to a widespread belief that the demand for donor organs could be satisfied if a greater proportion of available organs were donated for transplantation. Among reasons for the apparent poor retrieval rate of donor organs ${ }^{3}$ are lack of medical knowledge, such as of the criteria for diagnosing brain stem death; lack of experience of requesting organ donation; time constraints such as in discussion with relatives or in scheduling theatre time ${ }^{5}$; and shortage of staffed intensive care beds. Media coverage has implied that intensive care staff are insufficiently committed to organ donation and reluctant to approach relatives, but rebuttal has come from Chisholm on behalf of transplant units. ${ }^{6}$

The previous estimate by Jennett did not result from audit but from combining epidemiological data about head injuries and other conditions that commonly result in brain stem death together with the experience of brain stem death in three neurosurgical units: Glasgow, Cambridge, and Swansea. ${ }^{7}$ This patchwork of sources was extrapolated to provide a nationwide estimate. ${ }^{8}$ Moreover, and crucial to the supply of donor organs for transplantation, there is the difference between brain stem dead potential donors and brain stem dead actual donors. In 1980, for example, Wilson and Cast reported that various exclusions resulted in only $15(17 \%)$ of the 86 brain stem dead patients in their neurosurgical intensive care unit becoming actual donors. ${ }^{9}$ This was despite positive motivation towards organ donation.

To make a more accurate and up to date assessment, therefore, of how many brain stem deaths there are and of the potential increase in supply of actual donors a confidential audit of all deaths in intensive care (excepting coronary care and neonatal intensive care) was initiated by the Department of Health on the recommendation of the Hoffenberg working party. ${ }^{3}$

\section{Methods}

The audit started on 1 January 1989 in regional and special health authorities in England. Books of serially numbered audit forms were issued by the Department of Health to health authorities for completion in each intensive care unit. Three audit periods for 1989 were scheduled-January to March, April to June, July to December - and two in 1990. Liaison between regional and special health authorities, the Department 
Intensive care unit it. $=0$

\section{SECTION A}

1. Deceased SURNAME

2. Deceased FORENAME

3. Deceased hospital number

4. Date of DEATH (day/month/year)

5. Sex of deceased $(M=$ male, $F=$ female $)$

6. Age of deceased (in complete years)

7. Cause of death $1=$ cerebrovascular accident (haemorrhage or infarction) $2=$ head injury $\quad 4=$ other intracranial cause $3=$ brain tumour $\quad 5=$ extracranial cause

8. Was the patient breathing spontaneously

$$
\text { at the time of death? } \quad(1=\text { No, } 2=\text { Yes })
$$

IF YES, GO ON TO SECTIONB

9. Was brain stem death a possible diagnosis? $(1=\mathrm{No}, 2=\mathrm{Yes})$ IF NO, GO ON TO SECTION B

10. Was the patient discussed with the transplant team or co-ordinator?

Were the tests for brain stem death performed?

$$
(1=\text { No, } 2=\text { Yes })
$$$$
(1=\mathrm{No}, 2=\mathrm{Yes})
$$
IF NO, GOONTO SECTIONB

12. Did the patient fulfil brain stem death criteria before cessation of heart beat?

13. Was there a general medical contra-indication

$$
\text { to organ donation? } \quad(1=\mathrm{No}, 2=\mathrm{Yes})
$$

IF YES, PLEASE SPECIFY:

THEN GO ON TO Q19

PLEASE INSERT CARD AND PRESS FIRMLY USING BALL POINT PEN

\section{SECTION A continued}

14. Was the possibility of organ donation suggested to relatives?

\section{$0=$ no known relatives}

$1=$ organ donation was not discussed with (known) relatives

$2=$ request for organ donation was refused

$3=$ organs were offered but none was retrieved

$4=$ relatives consented and deceased became organ donor

15. Was organ donation prevented for logistical reasons? $\quad(1=$ No. $2=$ Yes $)$

IF YES, PLEASE SPECIFY

Which organs were suitablefortransplantation? ORGANS WHETHER WHETHER

Which organs were donated? SUITABLE DONATED

WHETHER SUITABLE

$1=$ not suitable
$2=$ yes, suitable

$9=$ not known

heart

kidneys $9=$ not know

WHETHERDONATED

$$
\begin{aligned}
1 & =\text { not offered } \\
2 & =\text { donated } \\
3 & =\text { offered, but not } \\
& \text { retrieved }
\end{aligned}
$$

\begin{tabular}{|c|c|}
\hline $\begin{array}{l}\text { Were corneas suitable for } \\
\text { transplantation? }\end{array}$ & $\begin{array}{l}1=\text { neither cornea was suitable } \\
2=\text { one or both corneas suitable } \\
9=\text { not known }\end{array}$ \\
\hline $\begin{array}{l}\text { Were corneas donated for } \\
\text { transplantation? }\end{array}$ & $\begin{array}{l}1=\text { not offered } \\
2=\text { donated } \\
3=\text { offered but not retrieved }\end{array}$ \\
\hline $\begin{array}{l}\text { Surname and position of person } \\
\text { completing form }\end{array}$ & $\begin{array}{l}1=\text { doctor } \\
2=\text { nurse } \\
3=\text { co-ordinator } \\
4=\text { other }\end{array}$ \\
\hline VAME & \\
\hline
\end{tabular}

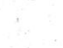

\section{SECTION B}

queries remained. The analysis file of 2853 forms therefore corresponds to about $92 \%$ of all deaths (estimated at 3085) in intensive care units in England between 1 January and 31 March 1989.

From 1 January to 31 March 1989, 163 donors of solid organs in England were notified to the United Kingdom Transplant Service. The audit analysis file comprises 148 donors of solid organs, and so intensive care units contributed an estimated $98 \%$ of solid organ donors in England ((148/163)/0.92).

Brain stem death was a possible diagnosis in only 407 (14\%) patients who died in intensive care units (see fig $3)$, but in $106(26 \%)$ of these cases brain stem function was not formally assessed. Tests for brain stem death were therefore performed in $300(11 \%)$ patients who died in intensive care units. The criteria for brain stem death were fulfilled before cessation of heart beat in 282 patients $(10 \%)$ and in the absence of any general medical contraindication to organ donation were fulfilled in only $234(8 \%)$ deaths in intensive care units. Figure 2 gives the reported general medical contraindications to organ donation and, for seven patients, logistic reasons (so called) that prevented donation. In 18 patients tests for brain stem death were performed but the criteria were not fulfilled; they constituted only $6 \%$ of 300 patients in whom tests for brain stem death were carried out.

The possibility of organ donation was discussed with relatives in 218 of 234 cases in which brain stem death was diagnosed in the absence of general contraindica-

FIG 1-Example of audit form sent to intensive care units (toned items show information not available to statistics unit)

of Health, and the Medical Research Council's biostatistics unit is through designated regional personnel.

Audit forms (fig 1) record $(a)$ regional and special health authority and intensive care unit alpha-numeric code (to ensure confidentiality these are assigned by regional liaison officers), date and cause of death, age and sex of deceased, and who completed the form; $(b)$ whether brain stem death was a possible diagnosis and if so was the patient discussed with the transplant team or coordinator, were the tests for brain stem death performed, and were the criteria fulfilled; $(c)$ whether, if the patient was brain stem dead, there was a general medical contraindication to organ donation and, if not, was organ donation suggested to relatives, which solid organs were suitable for donation, and which were donated; and $(d)$ whether corneas were suitable for donation and were they donated. Except for general medical contraindications to organ donation the audit form favours basic numbers over detailed answers but is designed so that basic answers readily identify cases for inclusion in supplementary inquiries.

\section{Results}

Of 278 intensive care units in England identified by regional and special health authorities, 258 (93\%) participated in the confidential audit of all deaths in intensive care from 1 January to 31 March 1989. Of 2863 forms received by 16 June 1989 (date of first analysis), only 10 were excluded from analysis because 
tions; consent for organ donation was given in $70 \%$ of cases $(95 \%$ confidence interval $64 \%$ to $76 \%$ ) - namely, in 152 out of 218 families with whom there was discussion. Refusal accounted for 66 cases. Organ donation was not discussed with (known) relatives in only 14 cases. Even when relatives agree to organ donation other considerations, including organ suitability, restricted permission for organ retrieval, and non-procurement of suitable organs, limit actual donation. Whereas restricted permission by otherwise consenting relatives resulted in no offer of suitable kidneys in only one case, there was no offer of suitable corneas from 42 patients whose relatives consented to other organ retrieval. Suitable kidneys were not procured in only two cases compared with 20 and 22 instances for liver and lungs respectively.

Figure 3 shows that $282(10 \%)$ patients who died in intensive care fulfilled brain stem death criteria, but solid organ donation was achieved in only 148 patients, $5 \%$ of all audited deaths. Of these, 143 were brain stem deaths and five were asystolic kidney donors. Thus half ( $51 \%$; $95 \%$ confidence interval $45 \%$ to $57 \%$ ) of brain stem dead potential donors became actual donors of solid organs. (Donation of corneas alone occurred in a further $63(2 \%)$ intensive care unit deaths.) Eighty nine $(60 \%)$ of the 148 solid organ donors donated solid organs of more than one type (for example, kidneys and heart). Asystolic kidney donation was requested in seven cases (from three regions) and was achieved for five patients, as above. Solid organ donations were reported as follows: 73 hearts, 145 kidney donors, 62 livers, 24 lung donors, and 20 pancreases. Corneal donation was reported in 123 cases.

In a high proportion $(1271 ; 45 \%)$ of deaths in intensive care units suitability of corneas for donation was "not known." In the one region where it was determined routinely corneas were suitable for donation in about three quarters of the region's 427 intensive care unit deaths. There was no offer, because no request was made or consent was not given, in 643 $(84 \%)$ of 766 patients who died in intensive care units and whose corneas were suitable for donation.

Figure 4 shows reported deaths in intensive care units per million residents by regional health authority (small area statistics from 1981 census) together with audit compliance. From 1 January to 31 March 1989 there were 61.5 reported deaths in intensive care units per million residents in regional health authorities. South and North East Thames health authorities had significantly $(p<0.0001)$ higher than expected numbers of deaths in intensive care units per million residents (122 and 79 respectively). The next highest rate was in North West Thames, and significantly lower rates were reported from bordering South West Thames, Wessex, Oxford, and East Anglia. Regional variations compatible with cross border transfer of patients suggest that it is more appropriate to compute expected numbers of solid organ donors relative to intensive care unit deaths than to resident or total population. The four Thames regions (resident population from 1981 census was 13.4 million; 1079 audited deaths in intensive care units) reported 36 solid organ donors. This is significantly fewer than the 56 expected $(p<0.01)$ if the all England rate of 5.2 solid organ donors per 100 deaths in intensive care units applied uniformly but is not extreme compared with 43 solid organ donors expected by resident population.

\section{Discussion}

Excellent response by colleagues in intensive care units has enabled us to analyse $92 \%$ of all deaths in intensive care in England from 1 January to 31 March 1989. We estimate that $98 \%$ of notified solid organ donors in England came from intensive care units. Only minority sources of solid organs are therefore excluded from the audit.

Brain stem death was a possible diagnosis in only
Haemophilus meningitis

Previous hypertension (severe) $)^{\star}$

Severe hypertension ${ }^{\star}$

Hypertension; anaemic hypoxia unresponsive to treatment ${ }^{\star}$

Hypertension

Hepatic failure

Prolonged hypotension (unrecoverable)

HIV infection; hepatitis $B^{\star}$

Heart failure; renal failure

Down's syndrome; congenital heart disease

Cirrhosis and metabolic defect; jaundice

Cerebral abscess; mitral valve replacement

Renal failure

Intracranial and extracranial sepsis

Disseminated intravascular coagulation

Cancer of oesophagus with metastases

Nephrotic syndrome; diabetic microangiopathy

Severe hypoxia secondary to acute respiratory distress

syndrome; sustained hypotension; terminal oliguria Pneumonia endocarditis

Age (81)

Pneumococcal septicaemia and meningitis

Hypotension due to intraabdominal injury ${ }^{\star}$

Age (74)^

Shigella infection

\section{Specified logistical reasons preventing some organ donation}

No suitable recipient identified for lungs/liver *

Not stated

Transplant team unable to come before heart beat stopped $^{\star}$

Not stated

Patient had breast cancer in $1983^{\star}$

Relatives very against donation

Patient arrested while waiting for theatre time ${ }^{\star}$

*Patient discussed with transplant team and coordinator

FIG 2-General medical contraindications to organ donation (48 cases) or donation prevented for logistical reasons (seven cases) 
$14 \%$ of patients who died in intensive care units, and we estimate about 1700 cases in England a year. (This estimate makes no allowance for seasonal variation, which is probably minor.) Brain stem death criteria were fulfilled before cessation of heart beat in $10 \%$ of patients who died in intensive care units, and we estimate about 1200 cases in England a year. In only 234 patients ( $8 \%$ of those dying in intensive care units), however, were brain stem death criteria fulfilled before cessation of heart beat and in the absence of any general medical contraindication to organ donation - that is, an estimated 1000 cases in England a year. Solid organ donation was achieved in 148 patients $(5 \%$ of all audited deaths), of whom five were asystolic kidney donors, giving an estimated 600 brain stem dead actual donors in England a year.

The above estimates of 1700 possible and 1200 confirmed cases of brain stem death are substantially lower than Jennett's 1981 estimate of 4000 brain stem deaths annually in Britain or 3400 annually in England. Possible explanations are methodological-arising from the difference between audit and extrapolation. Only minor inflation of our estimates would be warranted to account for patients who are ventilated outside intensive care units and whose deaths are thereby excluded from audit. Secondly, the incidence of brain stem death partly reflects the availability of mechanical ventilators and how often they are used for patients who might become brain stem dead-for example, those with intracranial haemorrhage. Intensive care facilities have always been at a premium, and the indications are that the pressure on staffed

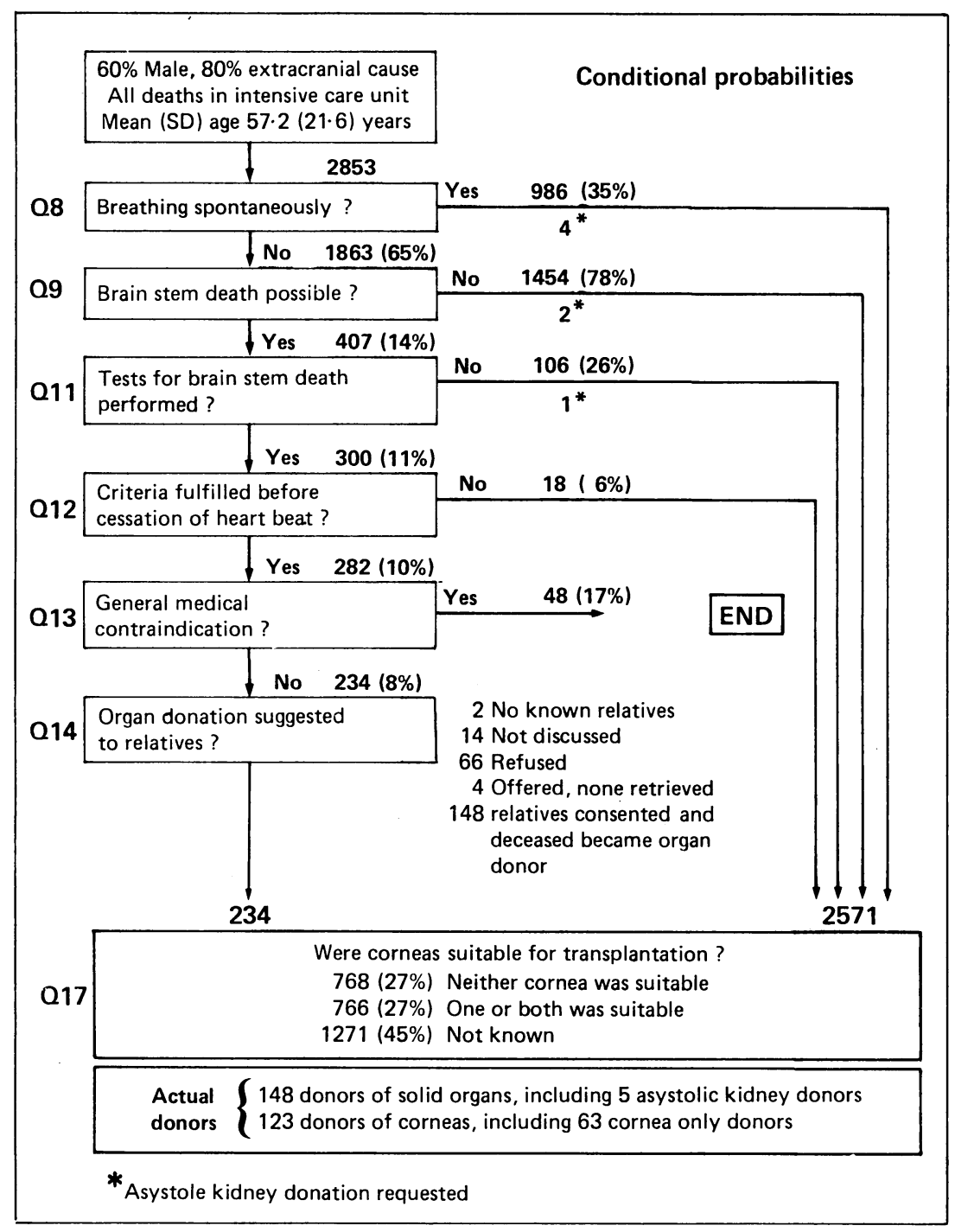

FIG 3-Flow chart of audit of deaths in intensive care units intensive care beds is increasing. In some instances, therefore, admission of a potential donor may be impossible. In our audit half $(95 \%$ confidence interval $45 \%$ to $57 \%$ ) of the confirmed cases of brain stem death in intensive care units became actual solid organ donors, which compares favourably with Wilson and Cast's $17 \%$ from one unit in 1978-9.9 Moreover, the audit does indicate a number of ways in which the retrieval rate of cadaver donor organs might be further improved.

An important finding was that formal testing of brain stem function was not performed in $26 \%$ of patients (95\% confidence interval $22 \%$ to $30 \%)$ for whom brain stem death was a possible diagnosis - that is, an estimated 450 patients a year in England. Unfortunately, the basic audit does not ascertain reasons for non-performance of tests for brain stem death in these patients, such as multiple organ failure, sepsis, intervening cardiac arrest, general medical contraindication, or negative motivation by intensive care unit staff. This could usefully be the subject of a supplementary inquiry as in some of these patients performance of tests for brain stem death, combined when necessary with more aggressive cardiovascular support, might have yielded extra donors. General medical contraindications precluded organ donation in $17 \%$ ( $12 \%$ to $22 \%$ ) of patients with confirmed brain stem death-that is, around 200 cases a year in England. Another source of extra donors is suggested by observing that some of the stated medical contraindications in figure 2 might not in fact have precluded donation of all organs, though brief comments may not, of course, convey the whole clinical picture. It is worth noting, for example, that extremes of age are often no longer considered to be contraindications. We recommend early consultation with the transplant team or coordinator to establish the suitability of organs for donation.

Consent to organ donation was given in $70 \%$ of cases (64\% to $76 \%$ ) when the possibility was suggested to relatives of a patient who fulfilled criteria for brain stem death and lacked any general medical contraindication to donation. This figure is remarkably similar to that reported in the British Kidney Patient Association's annual survey, conducted by Gallup, in March 1989, in which $70 \%$ of 1003 people surveyed said they would be willing to donate their kidneys after death. Our first audit period coincided with adverse publicity about kidney transplants from unrelated living Turkish donors, and media coverage is known to have both a positive and negative influence on the supply of organs for transplantation. The rate of refusal in the second audit period (April to June 1989), however, was also $30 \%$ ( 72 out of 242 families). Organ donation was not discussed with relatives of brain stem dead potential donors in only $6 \%$ of cases ( 14 out of 232 families $(3 \%$ to $9 \%))$. Introducing required request could therefore result in only a fairly small number of additional donors: about 40 a year in England, assuming a $30 \%$ refusal rate. The reason for no request, however, may have been that it was already quite clear that permission would not be given.

This audit suggests considerable potential to increase the numbers of corneal donors. Corneal suitability was recorded as "not known" in a high proportion (45\%) of all deaths in intensive care units in England, and there were only 123 corneal donors (4\% of all audited deaths). There was no offer because no request was made or consent was not given in 84 of 766 cases in which corneas were suitable for donation. Yet the Corneal Transplant Service eye banks are able to store corneal tissue in culture medium for up to 30 days for scheduled and emergency transplant operations throughout the United Kingdom. Discussion with transplant coordinators could provide the opportunity to organise corneal donation. This report 


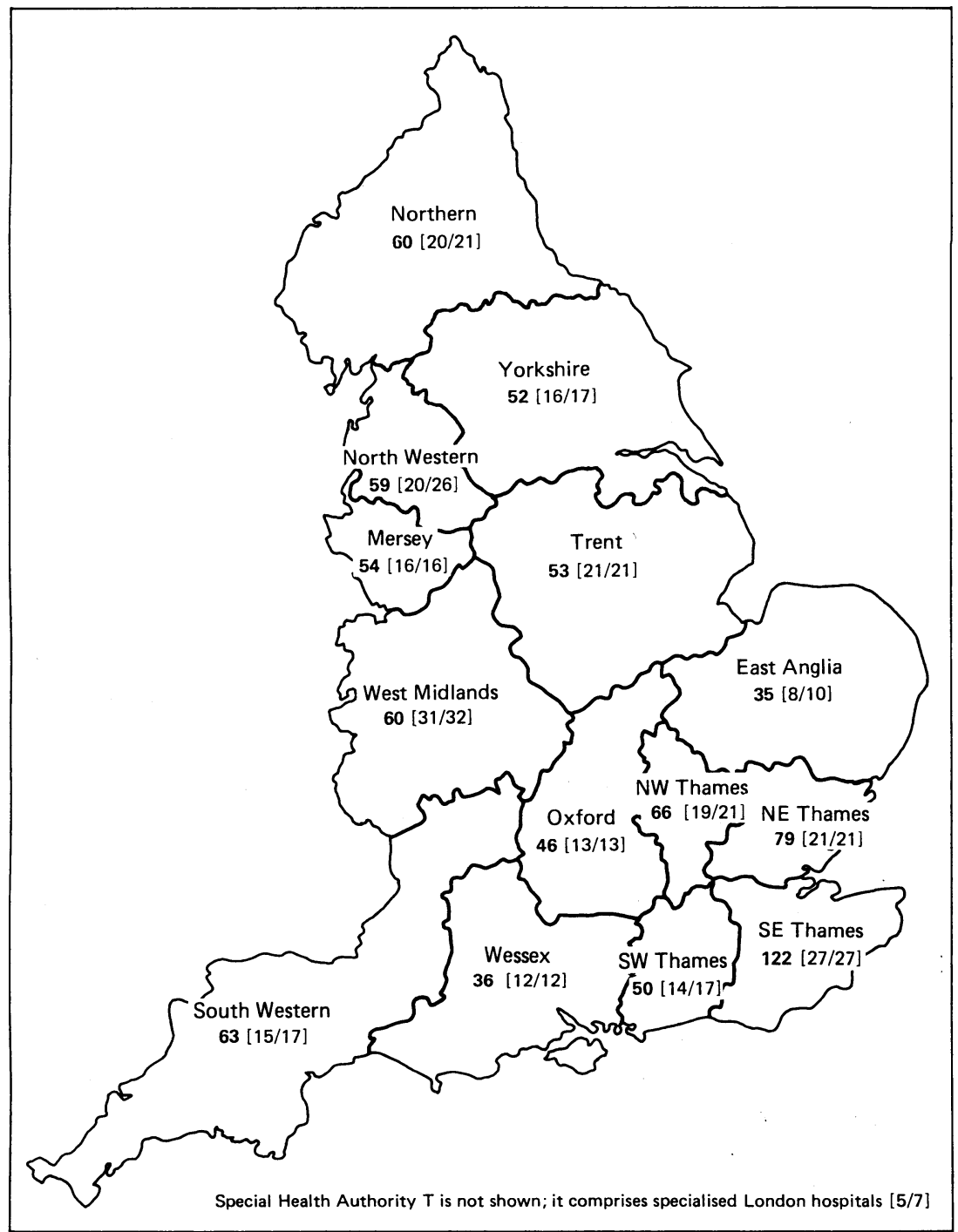

FIG 4-Reported numbers of deaths in intensive care units per million residents for regional health authorities in England [number of units responding/total number of units]

has shown important regional variation in deaths in intensive care units per million residents. In three Thames regions there was a significantly higher than expected number of deaths in intensive care units per million residents and corresponding low numbers in the surrounding four regions. These findings are compatible with cross border transfer of patients and suggest that it is more appropriate to compute expected numbers of solid organ donors relative to deaths in intensive care units, which are a more relevant denominator than a living population for comparison of rates of cadaver organ donation. In this way we have shown a deficit of reported solid organ donations in January to March in the four Thames regions, the reasons for which are at present unclear.

In conclusion, this three months' audit of all deaths in intensive care units in England puts the annual incidence of possible brain stem death (1700 cases) at half that estimated in 1981; the incidence of confirmed brain stem death ( 1200 cases) is about one third of the earlier estimate. Even if the proportion (51\%) of confirmed brain stem dead patients who become actual donors increased substantially, there would still be a shortage of cadaver donor organs for transplantation, as, for example, the projected need is for upwards of 2125 kidneys in England a year. These new figures have major implications for dialysis resources needed to achieve national targets for the treatment of end stage renal failure, prediction of which assumed that sufficient organs would be available to carry out the needed transplant operations. ${ }^{10}$ Chisholm has advocated more kidney transplantation from living related HLA identical donors. ${ }^{6}$ In cases of brain stem death earlier consultation with the transplant team and coordinator, particularly concerning medical contraindications, may increase the proportion of confirmed brain stem dead patients who become actual donors. Clinicians should perhaps be obliged to discuss all brain stem dead patients with their local transplant team or coordinator (required referral). This might also provide a useful means of increasing the number of corneal donors. Publicity (specific to different culture ${ }^{11}$ ) and public education could increase the proportion of potential donors in respect of whom relatives' consent is obtained. A supplementary inquiry has been initiated to establish the reasons for non-performance of tests when brain stem death is a possible diagnosis as this might represent a useful source of extra donors. It seems to be unusual for a request not to be made to the relatives of a suitable donor, and in many such instances refusal may in any case be likely. Additional cadaveric kidneys could be obtained from an increased use of asystolic donations.

Confidential audit of all deaths in intensive care is supported by a grant from the Department of Health. We thank particularly the doctors, nurses, coordinators, and others in intensive care units who have completed audit forms but whose identity, for reasons of confidentiality, is unknown to us. We are indebted to our regional liaison officers for the contacts that they have maintained with intensive care units (Dr Julie Bagnall, Sister Deirdre Barr, A J Cassidy, J Chapman, John S Chapman, R Collins, Nilli Lamakan, Christine Lyon, Jenny Wright, F Martin, M O'Donnell, Dr M A Pitman, Dr John Raison, Dr D Richardson, Dr M Rivlin, Frank Stockwell, Carol Hall, and Alan Waller). Mr Myc Riggulsford, public relations officer at United Kingdom Transplant Service, gave constructive advice on audit form content. Also consulted were the British Transplantation Society, British Organ Donor Society, Faculty of Anaesthetists, Intensive Care Society, Joint Consultants Committee, regional and district general managers, the Royal College of Physicians, Society of British Neurological Surgeons, and United Kingdom Transplant Service Management Committee.

1 UK Transplant Service. Annual report 1988. UK Transplant Service: Bristol, 1988.

2 Anonymous. Organ donation increasing in the United States. Eurotransplant Newsletter 1989;65:10-1.

3 Working Party on the Supply of Donor Organs for Transplantation. Report. London: HMSO, 1987. (Chairman: Sir Raymond Hoffenberg.)

4 Jennett B. Brain death. Br f Anaesth 1981;53:1111-9.

4 Jennett B. Brain death. Brf Anaesth 1981;53:1111-9. Grebenik CR, Hinds CJ. Management of the multiple organ donor. Brf Hosp
Med 1987 July: $62-5$.

6 Chisholm GD. Time to end softly softly approach on harvesting organs for transplantation. Br Med f 1988;296:1419-20.

7 Jennett B, Gleave J, Wilson P. Brain death in three neurosurgical units. $\mathrm{Br}$ Med F 1981;281:533-9.

8 Jennett $\mathrm{B}$, Hessett $\mathrm{C}$. Brain death in Britain as reflected in renal donors. $\mathrm{Br}$ Med f 1981;282:359-62.

9 Wilson PJE, Cast IP. Donor kidneys and restrictions on their supply. Br Med $\mathcal{F}$ 1980; 280:45-6.

10 Wood IT, Mallick NP, Wing AJ. Prediction of resources needed to achieve the national target for treatment of renal failure. Br Med f 1987;294:1467-70.

11 Perez LM, Schulman B, Davis F, Olson L, Tellis VA, Matas AJ. Organ donation in three major American cities with large Latino and black donation in three major American cities
populations. Transplantation 1988;46:553-7.

(Accepted 17 October 1989)

4

